\title{
UBER DIE ZERREISSUNG DER NABELSCHNUR DES SCHAFES
}

von

\author{
C. NAAKTGEBOREN UND A. P. M. WITMOND
}

\author{
Zoologisches Laboratorium der Universität von Amsterdam
}

\section{EINLEITUNG}

Bei den Mehrzahl der bisher untersuchten Wiederkäuer enthält der nach der Geburt am Bauch des Jungen zurückbleibende Rest des Nabelstranges keine Stümpfe der Blutgefäße. Bei dem natürlichen Riß der Nabelschnur werden die Gefäße innerhalb des Nabelringes zerrissen (Fischer, 1932; NAAKTGEBOREN, 1963). Dies sollte für das Schaf aber nicht zutreffen, denn nach den Angaben Meyers (1914) befinden sich im Nabelschnurrest am Bauch des Neugeborenen zwei Venenstümpfe von etwa $3 \mathrm{~cm}$ Länge. In der vorliegenden Arbeit haben wir die Nabelschnur des Schafes und deren Zerreißung genauer untersucht um die Beschreibung Meyers nachzuprüfen und die Verhältnisse beim Schaf mit den anderen schon untersuchten Tierarten vergleichen zu können. Zudem haben wir kinematographische Aufnahmen (Bildfrequenz 64 Bilder/Sekunde) gemacht, die uns einen genaueren Einblick in den Vorgang des Risses und in die Reihenfolge des Zerreißens der einzelnen Komponenten der Nabelschnur erlaubten, indem wir den Film mit 2 Bilder/Sek. vorführten, also um $32 \mathrm{mal}$ gedehnt. Wir sind der Meinung in dieser Weise zur Kenntnis der Zerreißung der Nabelschnur der Wiederkäuer wesentlich beitragen zu können, da die kinematographischen Zeitdehneraufnahmen eine genauere und zuverlässigere Beschreibung des Nabelstrangrisses gestatten als die bisher vorliegenden Beschreibungen.

\section{LITERATURÚBERSICHT}

Bei der Geburt findet eine Trennung von Plazenta und Frucht, die bis zu diesem Augenblick durch die Nabelschnur mit einander verbunden waren, statt. Die Durchtrennung der Nabelschnur kann entweder stattfinden indem die Schnur von der Mutter durchbissen wird (z.B. bei den Nagern, Landraubtieren und Affen) oder der Nabelstrang reißt spontan (z.B. bei den Walen und Huftieren). Beim spontanen Nabelschnurriß können wir Arten mit präformierten Riß- stellen und solche bei welchen die Nabelschnur an einer willkürlichen Stelle reißt (z.B. Schwein, Flußpferd und Pinnipedier), unterscheiden (NAaKTGEBoREN, 1963). Hier ist nur der spontane Riß von Bedeutung. Eine eingehende Beschreibung der verschiedenen Rißstellen, die bei den einzelnen Säugern bisher in der Literatur mitgeteilt wurden, findet sich bei NAAKTGEBonen (1963), weshalb wir es hier für richtig halten nur die wichtigsten Punkte hervorzuheben. Beim Pferd findet sich die präformierte Rißstelle gerade distal des Nabels. Alle Komponenten der Nabelschnur werden an dieser Stelle zerrissen. Ein Musculus sphincter umbilicalis ist hier vorhanden und verschliesst den Nabelstrangstumpf nach stattgefundenem Riß (Hauptmann, 1911; Fischer, 1932). Diese Befunde konnte NAAKTGEBOREN (1963) bestätigen am Przewalskipferd. Der Riß des Nabelstranges des Hausrindes geht ganz anders vor sich. Die Arterien werden in der Nähe der Harnblasenspitze und die Venen in dem Nabelring zerrissen. Der Urachus reißt bei der Harnblasenspitze. Die Amnionscheide der Nabelschnur reißt an einer willkürlichen Stelle, obwohl meistens zwischen dem Nabel und der Mitte der Nabelschnur. Die Arterien sind an ihrer Rißstelle sehr lose im Bindegewebe eingebettet und die Adventitia ist hier am dünnsten. Die Muskelschicht der Arterienwand enthält an der Rißstelle etwa $86 \%$ zirkulaire Muskelfasern, gegen nur 21\% im übrigen intraabdominalen und $35 \%$ im extraabdominalen Bereich. Die Venen reißen gerade distal der Vereinigungsstelle, wo sich auch die Struktur ändert. Die Venenwand wird hier nämlich dünner und die Venen liegen hier fest im Bindegewebe. Der Urachusriß an der Harnblasenspitze ist leicht verständlich, denn gerade hier geht die muskulöse Harnblase über in die hüllenartige Urachuswand (Frscher, 1932; NAAKTGEBOREN, 1963).

Bei den Säugern, deren Nabelschnuren an willkürlichen Stellen reißen (Schwein, Flußpferd) oder in der Nähe der Plazenta (Pinnipedier) oder deren Na- 
belschnur von der Mutter durchbissen wird (Affen), sind die Stellen, die der Rißstelle des Rindes entsprechen, nicht von besonderer Struktur. Bei den Walen konnten präformierte Rißstellen nachgewiesen werden. Die Ahnlichkeit mit den beim Hausrind festgestellten Verhältnissen ist groß. Nur reißt die Amnionscheide immer an derselben Stelle, die etwa dem Zustand beim Pferde entspricht (NAAKTGEBoren, 1963).

\section{MATERIAL UND METHODEN}

Das Material für unsere Untersuchungen wurde uns vom Amsterdamer Schlachthof großzügig zur Verfügung gestellt. Für die Mitarbeit der Verwaltung und des Personals des Schlachthofes sind wir zu großem Dank verpflichtet. Wir erhielten die Feten in uteris, also waren die Fruchthüllen und Nabelschnuren immer noch intakt. Ein Teil des Materials wurde für histologische Untersuchungen in Formalin 5\% fixiert. Zudem wurden Nabelschnurzerreißproben durchgeführt nach der schon beschriebenen Technik (NaAktgeboren, 1963; Seite 36). Da aber die Zugkraft, bei welcher die Nabelschnur zerrissen wird, viel kleiner ist als beim Rinde, wurde ebenfalls eine geänderte Methode angewandt. Der Fetus wurde dazu aufgehängt und an der Nabelschnur wurde ein Behälter befestigt, der allmählich mit Sand gefüllt wurde. Infolge des zunehmenden Gewichtes reißt die Nabelschnur. Nach dem Riß wird der Behälter mit Sand gewogen. Zur Beobachtung des Risses wurden Zeitdehneraufnahmen gemacht, wie schon oben erwähnt wurde. Das Alter der Feten wurde bestimmt nach Schmaltz (1921) und Malan und Curson (1936).

\section{DIE NABELSCHNUR DES SCHAFES}

\section{a Länge der Nabelschnur}

Im allgemeinen ist die Nabelschnur des Schafes kurz. Von 108 Schafsfeten, deren Nabelschnuren gemessen wurden, stellten wir Längen von 4 bis $18 \mathrm{~cm}$ fest. In 70 von den 108 Fällen befand sich die Länge zwischen 10 und $13 \mathrm{~cm}$. In Tabelle 1 und Abb. 1 sind die Angaben nach dem Alter der Frucht in Prozent der Scheitel-Steiß-Länge dargestellt worden. Hieraus ergibt sich einwandfrei eine relative Abnahme der Länge des Nabelstranges während der Tragzeit, so $\mathrm{daB}$ die Nabelschnur zur Zeit der Geburt kurz ist. Genau dasselbe wurde festgestellt beim Hausrind (NAaxtgeboren, 1963) und beim Finnwal (Slijper, 1960). Auf die für die uniparen Huftiere sehr große Bedeutung des Besitzes einer kurzen Nabelschnur wurde von $\mathrm{DE}_{\mathrm{E}} \mathrm{S} O \mathrm{O}$ (1947) hingewiesen. Eine längere
Tabelle 1. Relative Nabelschnurlänge der Schafsfeten nach dem Alter (Vergleiche Abb. 1).

\begin{tabular}{|c|c|c|}
\hline $\begin{array}{c}\text { Alter } \\
\text { in } \\
\text { Wochen }\end{array}$ & $\begin{array}{c}\text { Zahl } \\
\text { der } \\
\text { Feten }\end{array}$ & $\begin{array}{c}\text { Mittlere Nabelschnurlänge in \% } \\
\text { der } \\
\text { Scheitel-Steiß-Länge }\end{array}$ \\
\hline 10 & 2 & 54,6 \\
12 & 1 & 42,3 \\
14 & 4 & 50,6 \\
15 & 1 & 41,7 \\
16 & 22 & 45,4 \\
17 & 22 & 41,4 \\
18 & 10 & 38,4 \\
19 & 14 & 34,1 \\
20 & 11 & 31,4 \\
21 & 8 & 30,5 \\
22 & 7 & 26,0 \\
23 & 6 & 26,9 \\
\hline
\end{tabular}

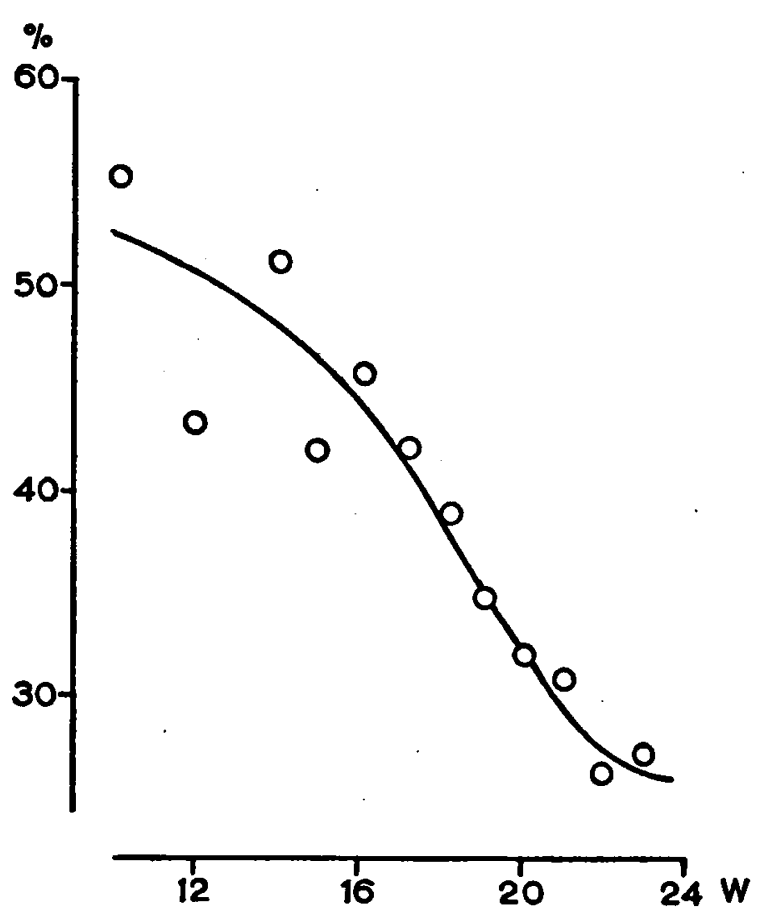

Abb. 1. Länge der Nabelschnur in Prozent der ScheitelSteiß-Länge des Schafsfetus, während der embryonalen Entwicklung.

Nabelschnur würde mit Leichtigkeit im Geburtswege vorgewölbt werden, soda $B$ die Abklemmungsgefahr wärend der Austreibung sehr groß wäre.

\section{b Bau der Nabelschnur}

In der Nabelschnur verlaufen zwei Venen, zwei Arterien und der Urachus, die von der Whartonsche Sulze umgeben sind. Die Nabelschnur ist von der Amnionscheide umgeben. Die Amnionscheide kann 

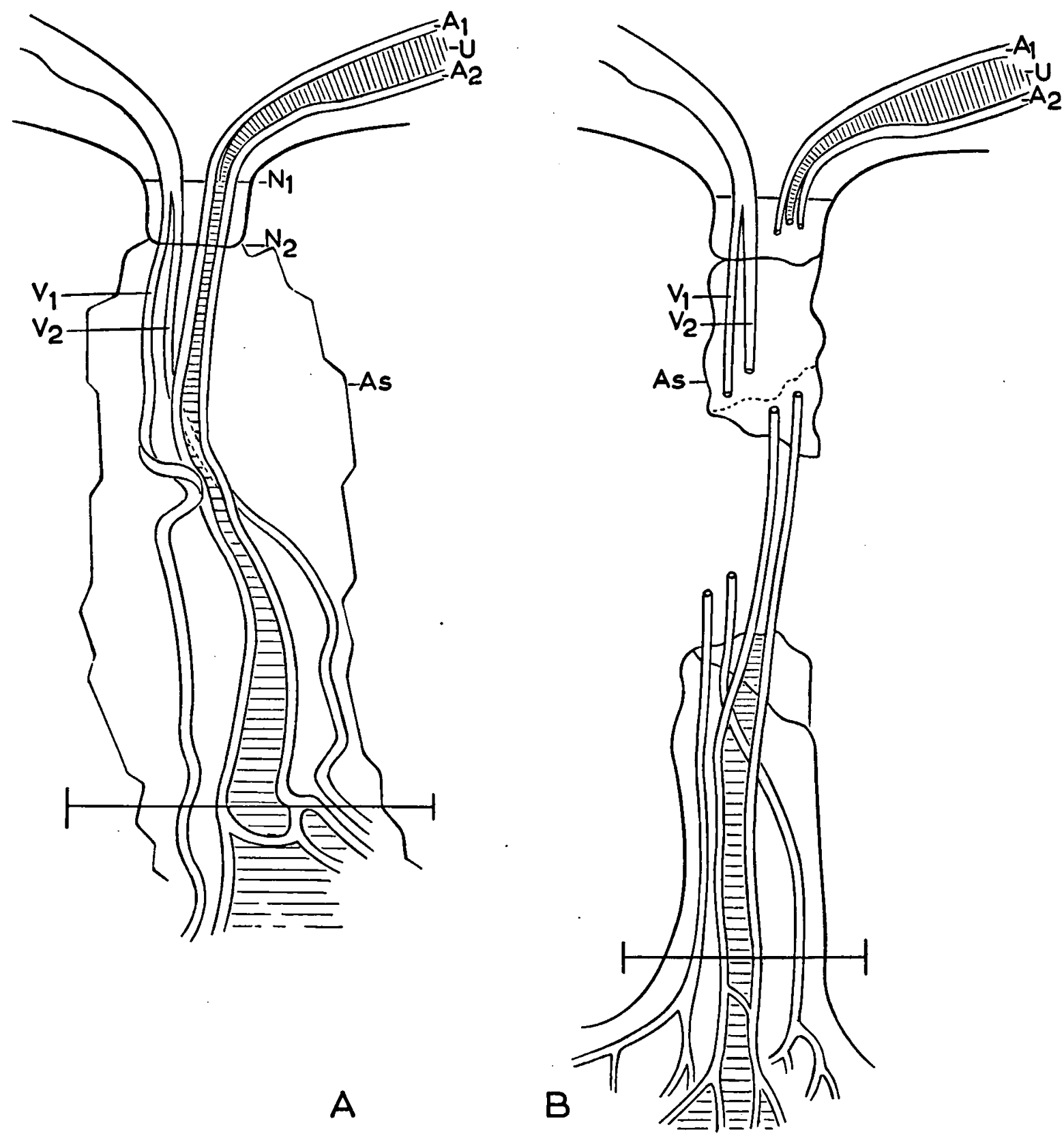

Abb. 2. Schematische Darstellung des Nabels und der Nabelschnur beim Schafe vor (A) und nach (B) dem Riß des Nabelstranges. $A_{1}, A_{2}$ : Nabelarterien, $U:$ Urachus, $V_{1}, V_{2}$ : Nabelvenen, As: Amnionscheide, $N_{1}:$ innerer Nabelring, $\mathrm{N}_{2}$ : äußerer Nabelring. Die quere Linie gibt die Ubergangsstelle zwischen Nabelschnur und Plazenta an.

mit gelblichen Wucherungen verschiedener Form besetzt sein. Diese Wucherungen haben eine der Haut entsprechende Struktur und wurden eingehend beschrieben von Schramm (1962) und NaAkTgeboREN und Zwillenserg (1961). Am äußeren Nabelring geht die Amnionscheide in die Haut über und bei der Verzweigung der Nabelgefäße in der Plazenta geht die Amnionscheide über in das freie Amnion, das ge- rade an dieser Stelle häufig sehr reich mit Amnionperlen besetzt ist.

Die Blutgefäße in der Nabelschnur verlaufen von dem Nabel zur Plazenta. In der gestreckten Nabelschnur verlaufen die Arterien vollkommen gerade. Die Venen sind in diesem Falle nicht gerade in ihrem Verlauf, sondern zeigen noch mehrere kleine Schlingen. Die eine Vene (Abb. 2, V2) biegt sich hinter den 
Arterien zur einen Plazentarhälfte, während die andere Vene (Abb. 2, Vl) vor den Arterien zum anderen Plazentarteil verläuft. In der letztgenannten Vene konnten wir fast immer eine sehr starke Schlinge nachweisen (Abb. 2). Nachdem man die Venen und Arterien auspräpariert hat, ist es möglich die Gefäße in gestreckter Lage genau zu messen. Es hat sich herausgestellt, daß die Venen immer länger sind als die Arterien und zwar gewöhnlich um $2 \frac{1 / 2}{2}$ bis $3 \mathrm{~cm}$. Wie unten noch anzuführen ist, ist diese Tatsache von entscheidender Bedeutung für den Verlauf des Nabelschnurrisses und für die Reihenfolge der am Riß beteiligten Komponenten des Nabelstranges.

\section{DAS ZERREISSEN DER NABELSCHNUR}

\section{a Der natürliche Nabelschnurriß}

Barclay (1946) beschreibt die Geburt des Schafes und nach seinen Angaben steht die Mutter während der Geburt. Die Nabelschnur wird dann infolge des Gewichtes des Jungen zerrissen. Am Nabel bleiben innerhalb des Amnionscheiderestes zwei Venenstümpfe von etwa $3 \mathrm{~cm}$ Länge zurück (MEYER 1914). Wenn die Mutter das Junge in liegender Haltung zur Welt bringt, wird die Nabelschnur während des Aufstehens der Mutter zerrissen. Bei einem totgeborenen Vicugnafohlen konnten wir den Nabelschnurrest untersuchen und auch hier waren die Venen außerhalb des Nabelringes zerrissen, und zwar etwa $2 \mathrm{~cm}$. Die Arterien waren im Abdomen gerissen.

\section{b Der Vorgang des Risses im Experiment}

Wir haben über fast ausgestragene und jüngere Feten verfügen können für die Durchführung des experimentellen Nabelscinnurrisses. Wir benutzten vorzugsweise die größten Feten, die also nahezu ausgetragen waren. Bei allmählichem Zug an der Nabelschnur wird diese zerrissen in einer Weise, die zu demselben Resultat führt wie der natürliche Riß während der Geburt. Am Fetus blieb nämlich ein Rest der Amnionscheide (As) mit zwei Venenstümpfe (Abb. 2B, Vl, V2) zurück, während die Arterien und der Urachus proximal des äußeren Nabelringes (N2) zerissen waren.

Die Zeitdehneraufnahmen ermöglichen eine genaue Beschreibung der Art und Weise des Nabelstrangrisses. Allmählich wird die ganze Nabelschnur etwas gespannt. Die Arterien sind ganz straff gespannt und infolge der immer zunehmenden Zugkraft werden sie schließlich zerrissen und zwar an einer ganz bestimmten Stelle, nämlich zwischen äußerem und innerem Nabelring (Abb. 2B). Der Urachus reißt wahrscheinlich zugleich mit den Arterien. Der ge- ringe Wandstärke des Urachus wegen ist dieser in den Filmaufnahmen nicht zu erkennen. Nachdem die Arterien zerissen sind, wird die Amnionscheide gespannt, was aus dem Film deutlichst hervorgeht. Bald aber reißt auch die Amnionscheide. Die Venen verlieren ihre Schlingen schon teilweise während des Spannens der Amnionscheide, aber nach deren ReiBen, werden sie ganz straff gezogen. Es stellte sich heraus, da $\beta$ die Venen sehr stark ausgereckt werden können, und häufig eine elastische Verlängerung von 2 bis $61 / 2 \mathrm{~cm}$ erfahren, gelegentlich sogar noch mehr, bevor sie reißen. Der Riß der Venen findet außerhalb des Nabels statt. Die Bauchwand bleibt starr und beteiligt sich also nicht aktiv am Riß. Der starre und derbe Rand des Nabels ist aber von Bedeutung für den Riß der Arterien, wie NaAkTgeboren (1963) schon für das Rind gezeigt hat und es liegen keine Anweisungen vor um anzunehmen, daß dies beim Schaf nicht auch der Fall sein sollte.

Ein sehr wichtiger Befund ist die genaue Feststellung des Fehlens der Elastizität während des Arterienrisses. Von einem Riß außerhalb des Nabels und einem Zurückschnellen in den Körper kann also nicht die Rede sein. NAAKTgEBoren (1963) hat dies schon auf Grund anatomischer Wahrnehmungen für das Rind angeführt, dies wird von den Zeitdehneraufnahmen bestätigt. Wohl werden die Arterien ein wenig nach distal gezogen (obwohl die Rißstellen noch innerhalb des Nabels bleiben), bevor der Riß stattfindet. Der Riß findet an einem Punkt, der sich vorher an derselben Stelle befindet wie der Stumpf nach vollendetem Riß, statt. Dies konnte einwandfrei nachgewiesen werden, indem wir den Nabel aufpräparierten und dann die Gefäße, die jetzt in ihrem ganzen Verlauf einzeln zu beobachten waren, zerrissen. Auch dieser Riß wurde zeitgedehnt gefilmt, denn selbst-

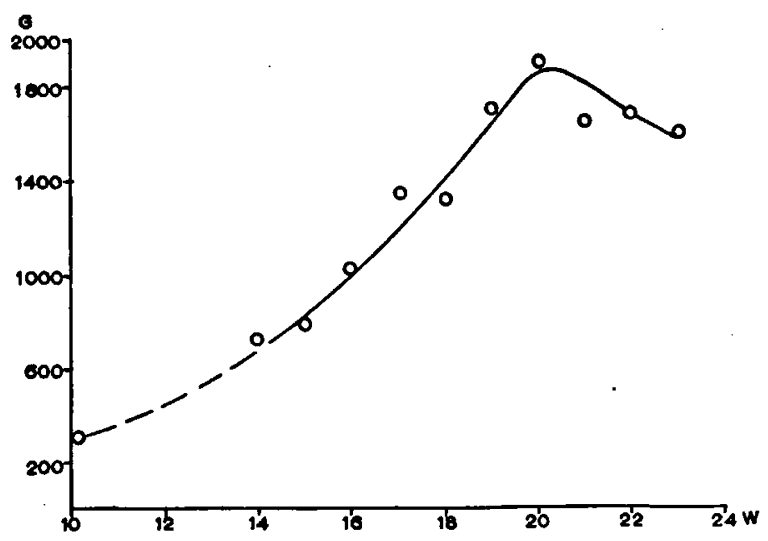

Abb. 3. Tragfähigkeit der Nabelschnur des Schafsfetus während der pränatalen Entwicklung in Gramm. Nach etwa 20 Wochen nimmt die Tragfähigkeit ab. 
verständlich kann man dies bei Betrachtung ohne weitere Hilfsmittel am Präparat nicht feststellen.

Zusammenfassend ist also zu sagen, daß beim Nabelschnurriß des Schafes die einzelnen Komponenten zerrissen werden immer in dieser Folge: 1. Arterien und Urachus. 2. Amnionscheide. 3. Venen. Die Arterien haben eine präformierte Rißstelle zwischen innerem und äußerem Nabelring und die Venen besitzen wahrscheinlich eine schwächere Stelle zwischen 0,5 und $5 \mathrm{~cm}$ distal vom Nabel, deren Lokalisation nicht immer genau dieselbe ist. Unten (Abschnitt 6) werden wir die Ergebnisse der mikroskopischen Untersuchung mitteilen.

Tabelle 2. Gewicht des Fetus und Tragfähigkeit der Nabelschnur. Man beachte die ziemlich großen individuellen Unterschiede.

\begin{tabular}{|c|c|}
\hline $\begin{array}{c}\text { Fetusgewicht } \\
\text { in Gramm }\end{array}$ & $\begin{array}{l}\text { Tragfähigkeit } \\
\text { in Gramm }\end{array}$ \\
\hline $\begin{array}{c}48 \text { und } 51 \\
400 \\
500 \\
600 \\
700 \\
800 \\
900 \\
1000 \\
1100 \\
1200 \\
1300 \\
1400 \\
1500 \\
1600 \\
1700 \\
1800 \\
1900 \\
2000 \\
2100 \\
2200 \\
2300 \\
2400 \\
2500 \\
2600 \\
2700 \\
2800 \\
2900 \\
3000 \\
3200 \\
3300 \\
3400 \\
3700 \\
4100 \\
4300 \\
4500 \\
4700 \\
5000 \\
5500 \\
5600\end{array}$ & $\begin{array}{l}253,285 \\
600 \\
700 \\
800,800,800, \\
800 \\
1100,1200 \\
800,1000,1300 \\
900,1000,1000,1000, \\
\quad 1100,1200 \\
1100 \\
1400 \\
1000,1300 \\
900 \\
1300,1500 \\
1200,1500 \\
1500.1500,1700 \\
800,1200,1300 \\
1300 \\
900,1600,1600,1700 \\
1300 \\
1300,2500 \\
1300,1700 \\
1200,1500 \\
1900 \\
1700 \\
1800 \\
1700,2300 \\
1200,1900 \\
1600,1900 \\
2200,2200,3000 \\
1400,1600,1700,2300 \\
1500 \\
1900 \\
1700 \\
1400 \\
1400 \\
1500 \\
1500 \\
2500 \\
1500 \\
\end{array}$ \\
\hline
\end{tabular}

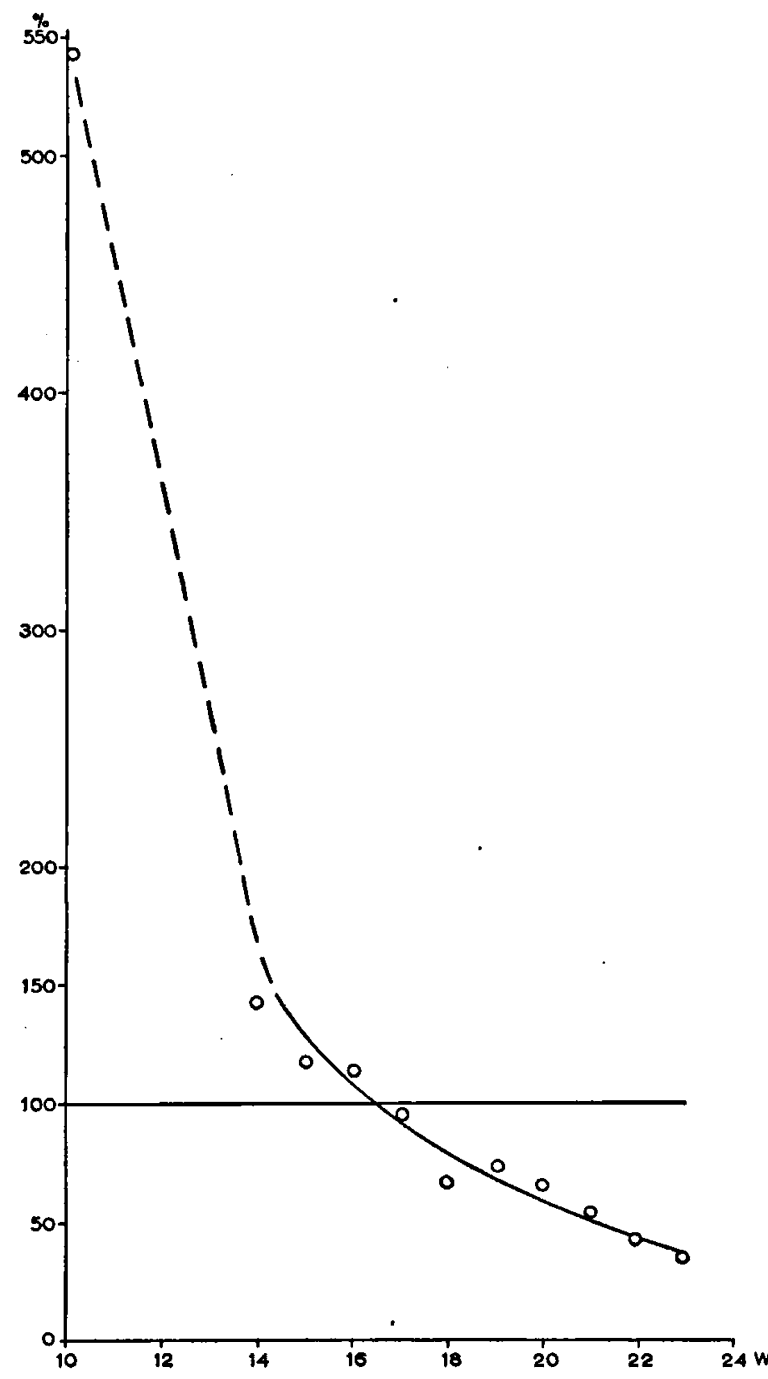

Abb. 4. Die Zugkraft, durch welche die Nabelschnur zerrissèn wird, in Prozent des fetalen Gewichtes nach dem Alter des Fetus. Zwischen 16 und 17 Wochen entspricht diese Kraft etwa dem Gewicht des Fetus.

\section{c Ursache des Zerreißens}

Während der embryonalen Entwicklung nimmt die Tragfähigkeit der Nabelschnur anfänglich zu, aber in den letzten Wochen kann man eine deutliche $\mathrm{Ab}$ nahme feststellen, wie aus Abb. 3 hervorgeht. In Abb. 4 sind dieselben Daten eingetragen, aber die Tragfähigkeit wurde nun umgerechnet in Prozent des Fetusgewichtes. Die Angaben der 10 Wochen alten $\mathrm{Fe}$ ten sind nicht zuverlässig, da die Nabelschnur nicht zerrissen wird, sondern die Nabelschnur wird aus dem Bauch des Fetus gezogen. Diese Art von Trennung entspricht also nicht den Verhältnissen bei der Geburt. In den Tabellen 2 und 3 sind alle Ergebnisse 
Tabelle 3. Die Tragfähigkeit der Nabelschnur in Prozent des Fetusgewichtes, nach dem Alter des Fetus.

\begin{tabular}{|c|c|c|}
\hline $\begin{array}{c}\text { Alter in } \\
\text { Wochen }\end{array}$ & $\begin{array}{c}\text { Tragfähigkeit der Nabelschnur } \\
\text { in \% des Fetusgewichtes }\end{array}$ & Mittelwert \\
\hline 10 & 527,559 & 543 \\
14 & $125,133,150,156$ & 141 \\
15 & 114 & 114 \\
16 & $77,85,89,100,100,104$, & 111 \\
& $105,132,133,145,150$ & \\
17 & $44,64,68,75,80,87,88$, & 91 \\
& $88,94,98,99,100,100$, & \\
18 & $110,114,117,120$ & 64 \\
19 & $45,50,57,62,69,82,85$ & 70 \\
20 & $59,63,67,68,76,77,82$ & 64 \\
& $41,42,59,65,65,67,69$, & \\
21 & 70,94 & 51 \\
22 & $44,47,50,52,63$ & 41 \\
23 & $30,42,51$ & 32 \\
\hline
\end{tabular}

der Nabelschnurzerreißproben notiert, während in den Kurven (Abb. 3 und 4) nur die Mittelwerte gezeichnet sind. Aus den Abbildungen 3 und 4 und den Tabellen 2 und 3 geht einwandfrei hervor, daß die Nabelschnur während der letzten sechs Entwicklungswochen nicht mehr imstande ist, das Gewicht des Fetus zu tragen. Die Ursache des natürlichen Nabelschnurrisses ist also das Eigengewicht des Jungen. Diese Befunde stimmen vollständig überein mit den Angaben NaAkTgeborens (1963) für das Hausrind.

\section{d Weitere Ergebnisse der Nabelschnurzerreißproben}

Neben den künstlich zerrissenen Nabelschnuren deren Risse gefilmt wurden haben wir noch 69 andere Experimente durchgeführt. Die Ergebnisse wurden zum Teil schon im vorhergehenden Abschnitt erwähnt, aber zudem konnten wir nach stattgefundenem $\mathrm{Ri} ß$ die Rißstellen genau untersuchen. Diese Befunde stimmen mit den schon in Abschnitt $5 \mathrm{~b}$ beschriebenen Tatsachen überein. Auf einige Punkte soll hier noch etwas tiefer eingegangen werden. In allen Fällen wurden die Arterien im Nabel zerrissen. Ein Zurückschnellen ins Abdomen haben wir nicht beobachtet. Auch wenn der Nabel vorher geöffnet ist, und man also in der Lage ist die RiBstelle zu markieren, stellt sich heraus, daß die Arterien genau dort reißen, wo man nachher die Stümpfe findet. Zudem haben wir die Reste der Arterien nach dem Riß gemessen in 68 Fällen. Es hat sich herausgestellt, daß die Arterienreste am Nachgeburtsteil im Mittel gleich lang waren als die Nabelschnuren, weshalb hier nicht die Rede sein kann von einem $\mathrm{Riß}$ außerhalb des $\mathrm{Na}$ bels. Beim Schaf finden sich die Arterienstümpfe zwischen innerem und äußerem Nabelring (Abb. 2B), während sie beim Rind zur Höhe der Harnblasenspitze liegen (NaAktgeboren, 1963) und beim Pferd gerade distal des Nabels (Hauptuann, 1911).

Die Amnionscheide des Nabelstranges wird an einer willkürlichen Stelle zerrissen. Die Länge des Restes hängt nicht zusammen mit der Länge des ganzen Nabelstranges. Meistens ist der Rest, der an dem Fetus zurückbleibt, kürzer als die halbe Nabelschnur.

Die Venen reißen ausserhalb des äußeren Nabelringes. Tabelle 4 zeigt einige Befunde des experimentellen Nabelschnurrisses bei Feten des letzten Entwicklungsmonates.

Tabelle 4

\begin{tabular}{|c|c|c|c|}
\hline \multirow{2}{*}{$\begin{array}{c}\text { Alter } \\
\text { in } \\
\text { Wochen }\end{array}$} & \multirow{2}{*}{$\begin{array}{c}\text { Zahl } \\
\text { der } \\
\text { Feten }\end{array}$} & \multicolumn{2}{|c|}{ MITTELWERTE } \\
\hline & & $\begin{array}{c}\text { Länge der } \\
\text { Nabelschnur }\end{array}$ & $\begin{array}{c}\text { Länge des } \\
\text { Venenrestes }\end{array}$ \\
\hline $\begin{array}{l}20 \\
21 \\
22 \\
23\end{array}$ & $\begin{array}{l}9 \\
5 \\
3 \\
5\end{array}$ & $\begin{array}{l}12,1 \mathrm{~cm} \\
10,9 \mathrm{~cm} \\
13,0 \mathrm{~cm} \\
11,4 \mathrm{~cm}\end{array}$ & $\begin{array}{l}2,4 \mathrm{~cm} \\
2,5 \mathrm{~cm} \\
2,7 \mathrm{~cm} \\
4,9 \mathrm{~cm}\end{array}$ \\
\hline
\end{tabular}

Die präformierte Rißstelle der Venen liegt also distal des Nabels, ist aber nicht so genau lokalisiert wie die der Arterien. Während des Reißens werden die Venen elastisch gereckt, aber nach dem Riß retrahieren sie bis zu ihrer ursprünglichen Länge. Beim Zerreißen einiger auspräparierten Venen konnten wir eindeutig feststellen, daß die elastische Verlängerung am stärksten stattfindet in der Nähe der Rißstelle. $\mathrm{Da}$ die Venen also eine präformierte Rißstelle besitzen liegt auf der Hand. Beim Rind reißen sie einfach bei der Vereinigung der beiden Nabelvenen (Fischer, 1932). Dasselbe wurde für mehrere Wiederkäuer und die Wale festgestellt von NAAKTGEBoREN (1963).

\section{MIKROSKOPISCHE STRUKTUR DER RISSTELLEN}

Genau wie beim Rind liegen auch beim Schaf die Nabelarterien im Nabel sehr lose eingebettet, während die Venen sehr fest mit der Umgebung verbunden sind. Die Adventitia der Arterienwand ist nur im intraabdominalen Bereich gut entwickelt (Abb. 5), während sie an der Rißstelle und im extraabdominalen Teil nur eine unbedeutende Schicht darstellt (Abb. 6 bis 9). BarcLAY (1946) berichtet ebenfalls, daß die Adventitia an der Rißstelle kaum entwickelt ist. Dasselbe wurde von NAAKTGEBoren (1963) für das Rind beschrieben. Die Muskulatur der Arterien ist an der Rißstelle ganz besonders ausgebildet. Hier 


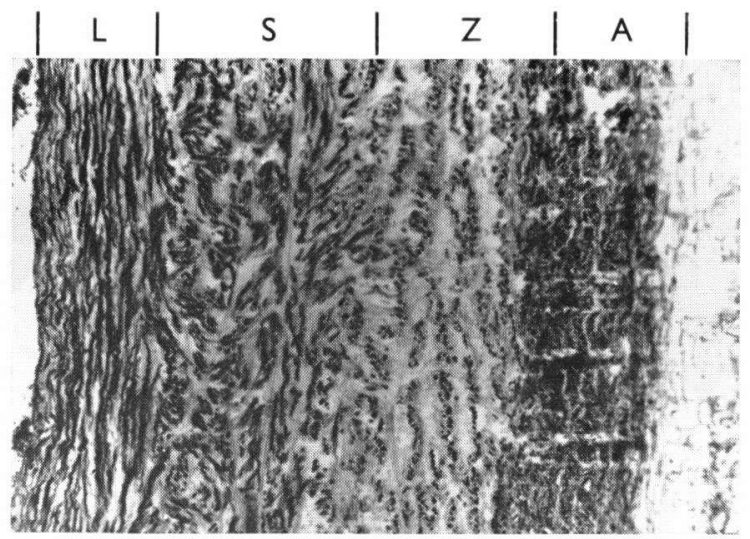

Abb. 5

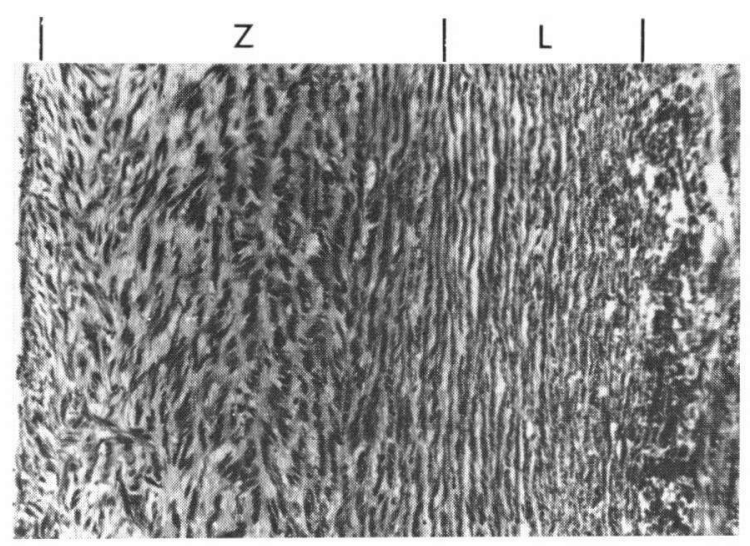

Abb. 6

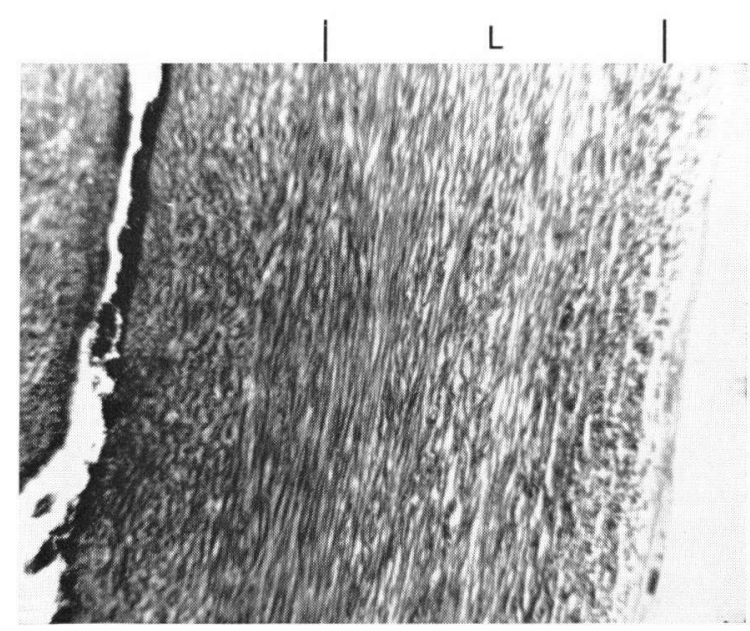

Abb. 7

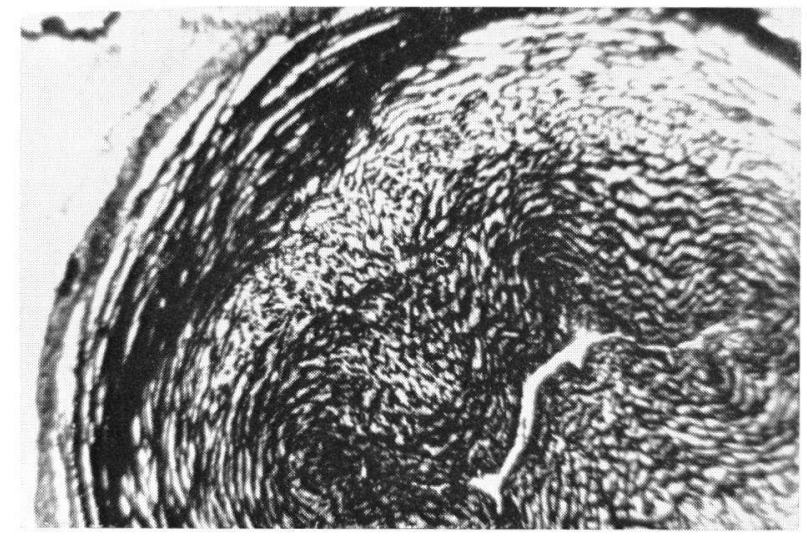

Abb. 8

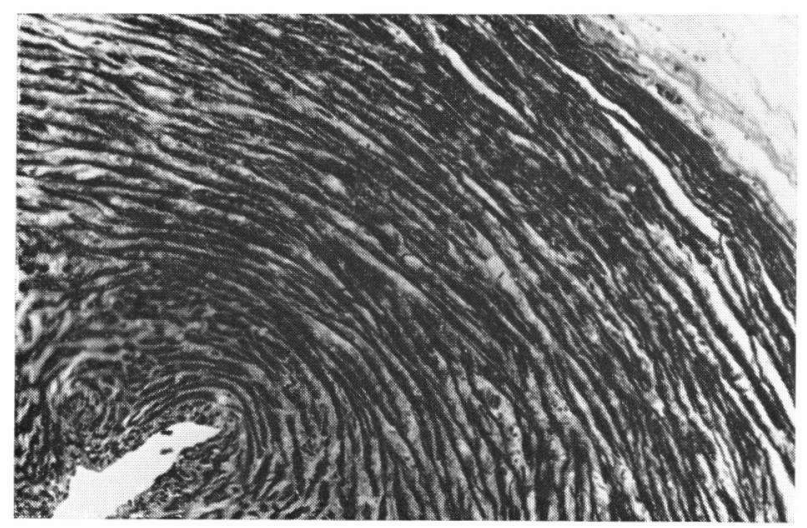

Abb. 9

Abb. 5. Längsschnitt durch die Wand der intraabdominalen Teiles der Nabelarterie. Die Adventitia (A) ist gut entwickelt. Die Muskelfasern verlaufen spiralig (S), zirkulair (Z) und in der Längerichtung $(L)$.

Abb. 6. Längsschnitt durch die Wand der Nabelarterie an der Rißstelle. Die Adventitia ist kaum entwickelt. Die Muskelfasern sind zum größten Teil zirkulair angeordnet (Z). L: Lïngsfasern.

Abb. 7. Längsschnitt durch die Wand der Nabelarterie in der Nabelschnur. Die Muskelfasern verlaufen hauptsächlich longitudinal $(L)$.

Abb. 8. Querschnitt durch die Nabelarterie, gerade distal des Nabels. Nur im periferen Bereich sind gut ausgebildete zirkulaire Muskelfasern vorhanden.

Abb. 9. Querschnitt durch die Nabelarterie an der Rißstelle. Fast alle Muskelfasern verlaufen zirkulair. 
findet man zirkulaire Fasern in einer überherrschenden Menge, während die Längsfasern nur spärlich vorhanden sind (Abb. 6). In den übrigen Teilen des Gefäßes bilden die Längs- und Spiralfasern den Hauptbestandteil der Wand (Abb. 5,7 und 8). Die Behauptung BARCLAYs (1946), daß alle Längsfasern an der Rißstelle fehlen, konnten wir nicht bestätigen, wie einwandfrei aus den Abbildungen 6 und 9 hervorgeht. Es liegt auf der Hand, daß an der Stelle wo wenig Längsfasern vorhanden sind, die Wand die geringste Zugfestigkeit besitzt und daß deshalb dort der Riß stattfindet. Die Rißstelle der Nabelarterien des Rindes hat etwa die gleiche Struktur (NAAKTgEBOREN, 1963).

In den Venen konnten wir infolge einer Abnahme der Längsmuskulatur eine Verjüngung der Wand um etwa $20 \%$ beobachten. Diese Verjüngung der Wand erklärt, daß der RiB hier stattfindet.

\section{VERGLEICHENDE BEMERKUNGEN}

Die präformierten Rißstellen der einzelnen Nabelschnurkomponenten können sich, abhängig von der Tierart, alle an derselben aber auch an verschiedenen Stellen befinden. Beim Pferde reißen alle Teile der Nabelschnur gerade distal des Nabels (HauptManN, 1911; Fischer, 1932). Beim Rind reißen die Venen im Nabel und die Arterien und der Urachus in der Bauchhöhle. Nur die Amnionscheide wird distal des Nabels zerrissen (NAAKTgEboREN, 1963). Beim Schaf werden der Urachus und die Arterien im Nabel und die Venen 2 bis $3 \mathrm{~cm}$ distal des Nabels zerrissen. Dasselbe wurde beim Vicugna (Lama vicugna) festgestellt. Auch die Amnionscheide reißt in einiger Entfernung vom Nabel. In allen diesen Fällen ist ausnahmslos für die Gefäße eine anatomisch nachweisbare Rißstelle gefunden worden. Eine morphologisch besondere Stelle ist nicht nachweisbar beim Flußpferd und beim Schwein deren Nabelschnuren an willkürlichen Stellen zerrissen werden. Eine solche auffällig gebaute Stelle fehlt ebenfalls beim Men- schen und bei den Affen, deren Nabelstränge bei der Geburt nicht spontan reißen (NAakTgEBonEN, 1963). Die Wale haben gut ausgebildete Rißstellen, die innerhalb des Nabels liegen. Es ist von größtem Interesse, daß Herr Kollege W. L. van UTRECHT in der Lage gewesen ist, Zeitdehneraufnahmen vom Riß der Nabelschnur eines Seiwales (Balaenoptera borealis) zu machen. Die Aufnahmen zeigen einwandfrei, daß die Amnionscheide wenig distal des Nabels reißt, aber da $B$ alle anderen Komponenten im Abdomen zerissen und nachher nach außen gezogen werden, was die anatomischen und histologischen Befunde NAAKTGEBORENs (1963) völlig bestätigt. Zusammenfassend ist also zu sagen, daß Art und Weise des Nabelschnurrisses anatomisch bedingt sind.

\section{ZUSAMMENFASSUNG}

An Hand von Zeitdehneraufnahmen wird der Riß des Nabelstranges beim Schaf beschrieben. Zuerst reißen die Arterien und der Urachus, dann die Amnionscheide und zuletzt werden die Venen zerissen. Diese Reihenfolge hängt mit den anatomischen Verhältnissen in der Nabelschnur zusammen. Die Venen sind nämlich länger als die Arterien und weisen in ihrem Verlauf mehrere größere und/oder kleinere Schlingen auf. Die Arterien reißen im Nabel. An dieser präformierten Rißstelle ist die Adventitia rückgebildet und besteht die Muskelschicht hauptsächlich aus zirkulairen Fasern, während an den anderen Stellen des Gefäßes die Längsfasern den Hauptteil der Wand bilden. Die Amnionscheide reißt an einer willkürlichen Stelle. Die Venen reißen etwa $3 \mathrm{~cm}$ distal des Nabels. An dieser Stelle verjüngt sich ihre Wand um etwa 20\%. Die Befunde werden mit den Verhältnissen bei anderen Tieren verglichen.

Es hat sich herausgestellt, daß die anatomischhistologischen Befunde bei allen bisher untersuchten Tierarten in Úbereinstimmung sind mit der Art des Zerreißens und mit der festgestellten Rißstelle nach der natürlichen Geburt.

\section{LITERATUR}

Barclay, A. E., K. J. Frankin, and M. M. L. Prichard: The foetal circulation and cardiovascular system and the changes that they undergo at birth. Blackwell Scientific Publications. Ltd Oxford. 1946.

Fischen, J.: Uber den Nabel des Kalbes sowie einiger anderer Haustiere mit besonderer Berücksichtigung seines Verhaltens bei der Geburt. Zeitschr. Anat. Entwicklungsgesch. $97,535,1932$.
Hauptmann, E.: Uber den Bau des Nabelstranges beim Pferde mit besonderer Berücksichtigung der natürlichen Rißstelle.

Zeitschr. Anat. Entwicklungsgesch. Seite 103, 1911.

Malan, A. P. and H. H. Curson: Further observations on the bodyweight and crown-rump length of Merino foetuses. Onderstepoort Journ. of Vet. Sci. and Animal Ind. 7 (1), 239, 1936. 
MEYER, A. W.: Some observations and considerations on the umbilical structures of the newborn. Am. Journ. Obstr. and Dis. Wom. 69, 214, 1914.

NaAkTgeboren, C.: Untersuchungen über die Geburt der Säugetiere. Bijdr. Dierkunde 32, 1, 1963. Diss. Amsterdam 1963.

NaAktgeboren, C. und H. H. L. Zwillenberg: Untersuchungen über die Auswiichse am Amnion und an der Nabelschnur bei Walen und Huftieren, mit besonderer Berücksichtigung des europäischen Hausrindes. Acta morphol. Neerl.-Scand. 4 (1), 31, 1961.
Schmaltz, R.: Das Geschlechtsleben der Haussäugetiere. Schoetz-Berlin 3. Aufl. 1921.

Schramm, B.: Les deux revêtements du cordon umbilical. Arch. Anat. Histol. Embryol. norm. et exp. 14, 35, 1962. SirJPER, E. J.: Die Geburt der Säugetiere. In Kükenthals Handbuch der Zoologie 8. Band, 25. Lief. 9 (9), 1, 1960.

SNOO, K. DE: Het probleem der menschwording in het licht van de vergelijkende verloskunde. Bohn-Haarlem 2. Aufl. 1947. 